\title{
SMALL ZEROS OF ADDITIVE FORMS IN MANY VARIABLES ${ }^{1}$
}

\author{
BY
}

\author{
WOLFGANG M. SCHMIDT
}

\begin{abstract}
It is shown that if $s$ is large as a function of $k$ and of $\varepsilon>0$, then the diophantine equation $a_{1} x_{1}^{k}+\cdots+a_{s} x_{s}^{k}=b_{1} y_{1}^{k}+\cdots+b_{s} y_{s}^{k}$ with positive coefficients $a_{1}, \ldots, a_{s}, b_{1}, \ldots, b_{s}$ has a nontrivial solution in nonnegative integers $x_{1}, \ldots, x_{s}, y_{1}, \ldots, y_{s}$ not exceeding $m^{(1 / k)+e}$, where $m$ is the maximum of the coefficients.
\end{abstract}

1. Introduction. A fairly direct application of the circle method shows that an equation

$$
a_{1} x_{1}^{k}+\cdots+a_{s} x_{s}^{k}=0
$$

where the coefficients $a_{1}, \ldots, a_{s}$ are not all of the same sign has a nontrivial solution in nonnegative integers $x_{1}, \ldots, s_{s}$, provided only that $s \geqslant c_{1}(k)$. (See, e.g., Davenport and Lewis [3], or Davenport [2].) As for the size of these solutions, it was shown by Pitman [6] that if the coefficients are as above, and each nonzero, and if $s \geqslant c_{2}(k)$ where $c_{2}(k)$ is explicitly given, then for given $\varepsilon>0$ there is a nontrivial solution in nonnegative integers with

$$
\left|a_{1} x_{1}^{k}\right|+\cdots+\left|a_{s} x_{s}^{k}\right|<c_{3}(k, \varepsilon)\left|a_{1} \cdots a_{s}\right|^{k+\varepsilon} .
$$

(Actually Pitman does not require the solutions to be nonnegative, hence for odd $k$ allows the coefficients to be of arbitrary signs. But the result quoted is an immediate outcome of her method.) In particular, for $s \geqslant c_{2}(k)$ there is a solution with

$$
\max \left(x_{1}, \ldots, x_{s}\right)<c_{4}(k) m^{c_{s}(k)}
$$

where $m=\max \left(\left|a_{1}\right|, \ldots,\left|a_{s}\right|\right)$.

Under suitable conditions, and if $s$ is very large, the estimate (1.2) may be considerably improved. Birch [1] combined Pitman's results with ideas contained in Linnik's elementary solution [4], [5] of Waring's problem to show that if $k$ is odd and if $s \geqslant c_{6}(k, \varepsilon)$, then (1.1) has a nontrivial solution in

Received by the editors October 5, 1977.

AMS (MOS) subject classifications (1970). Primary 10B30, $10 \mathrm{~J} 10$.

${ }^{1}$ Written with partial support from NSF MCS 75-08233 A02.

(C) 1979 American Mathematical Society 0002-9947/79/0000-0055/\$04.50 
integers $x_{1}, \ldots, x_{s}$, which may be of arbitrary sign, and which have

$$
\max \left(\left|x_{1}\right|, \ldots,\left|x_{s}\right|\right)<c_{7}(k, \varepsilon) m^{(1 / k)+\varepsilon} .
$$

This estimate is probably not the best possible. If the right-hand side of (1.3) could be improved to $c_{8}(k, \varepsilon) m^{\varepsilon}$, it would have the important consequence that a form of odd degree $k$ with real coefficients in enough variables can be made arbitrarily small for suitable (not all zero) integer values of the variables (see the remark in Birch [1]). For certain other applications in diophantine approximation, it is desirable to have a version where $k$ may be even as well as odd, and where each variable is of a prescribed sign.

Theorem. Suppose $s \geqslant c_{9}(k, \varepsilon)$, and suppose $a_{1}, \ldots, a_{s}, b_{1}, \ldots, b_{s}$ are positive integers. Then the equation

$$
a_{1} x_{1}^{k}+\cdots+a_{s} x_{s}^{k}=b_{1} y_{1}^{k}+\cdots+b_{s} y_{s}^{k}
$$

has a nontrivial solution in nonnegative integers $x_{1}, \ldots, x_{s}, y_{1}, \ldots, y_{s}$ having

$$
\max \left(x_{1}, \ldots, x_{s}, y_{1}, \ldots, y_{s}\right) \leqslant m^{(1 / k)+\varepsilon},
$$

where

$$
m=\max \left(a_{1}, \ldots, a_{s}, b_{1}, \ldots, b_{s}\right) .
$$

This is stronger than Birch's result even if $k$ is odd. The estimate is essentially best possible, for every nontrivial solution of

$$
a\left(x_{1}^{k}+\cdots+x_{s}^{k}\right)=b\left(y_{1}^{k}+\cdots+y_{s}^{k}\right)
$$

with coprime positive $a, b$ has $x_{1}^{k}+\cdots+x_{s}^{k} \geqslant b$ and $y_{1}^{k}+\cdots+y_{s}^{k} \geqslant$ $a$, whence

$$
\max \left(x_{1}, \ldots, x_{s}, y_{1}, \ldots, y_{s}\right) \geqslant c_{10}(k, s) m^{1 / k}
$$

where $m=\max (a, b)$. But it is conceivable that the Theorem holds with $c_{9}(k, \varepsilon)$ replaced by some $c_{9}^{\prime}(k)$, and the conclusion replaced by $\max \left(x_{1}, \ldots, x_{s}, y_{1}, \ldots, y_{s}\right) \leqslant c_{11}(k, \varepsilon) m^{(1 / k)+\varepsilon}$. The constant $c_{9}(k, \varepsilon)$ obtainable by our method is computable but very large.

Our proof is similar to Birch's in that we reduce the problem to that of finding solutions of

$$
a_{1} x_{1}^{k}+\cdots+a_{s} x_{s}^{k}-\left(b_{1} y_{1}^{k}+\cdots+b_{s} y_{s}^{k}\right)=z
$$

with very small $z$. But we shall employ the circle method instead of elementary estimates à la Linnik.

Our Theorem is applied by Schlickewei [7] to obtain a result about small values of indefinite diagonal forms with real coefficients.

\section{An inductive argument.}

Proposition 1. Let $\lambda \geqslant 1 / k, \varepsilon>0$ and $s \geqslant c_{12}(k, \lambda, \varepsilon)$. Let $a_{1}, \ldots, a_{s}$, $b_{1}, \ldots, b_{s}$ be as in the Theorem. Then (1.4) has a nontrivial solution in 
nonnegative integers $x_{1}, \ldots, x_{s}, y_{1}, \ldots, y_{s}$ with

$$
\max \left(x_{1}, \ldots, x_{s}, y_{1}, \ldots, y_{s}\right) \leqslant m^{\lambda+\varepsilon} .
$$

The case $\lambda=1 / k$ is the Theorem. Moreover, since the truth of the proposition for a particular value of $\lambda$ implies its truth for every $\lambda^{\prime}>\lambda$, the proposition is in fact equivalent to the Theorem.

It will suffice to prove the proposition when $m$ is large, say $m \geqslant c_{13}(k, \lambda, \varepsilon)$. For if $m<c_{13}$ and if $s$ is large, then the $a_{i}$ will assume the same value $a$ at least $m$ times, and the $b_{i}$ will assume the same value $b$ at least $m$ times, so that $a$ occurs at least $b$ times and $b$ occurs at least $a$ times, and from this one can construct a solution of the equation consisting of zeros and ones only. Proposition 1 is true for some values of $\lambda$ : By Pitman's estimate (1.2) it is true for $\lambda>c_{5}(k)$. Because of the $\varepsilon>0$ in the formulation of the proposition, the set of numbers $\lambda$ (this set depends only on $k$ ) for which the proposition holds is closed. Thus to prove Proposition 1 (and hence the Theorem), it will suffice to prove the following ${ }^{2}$

"INDUCTIVE AsSERTION." If $\lambda>1 / k$ and if the proposition is true for $\lambda$, then it is true for some $\lambda^{\prime}<\lambda$.

In what follows, $\lambda$ will be a fixed number $>1 / k$ for which the proposition holds. Pick $\mu$ so small that

$$
1 / k+6 c_{5}(k) \mu+20 \mu<\lambda \text { and } 22 k \mu<1,
$$

and put

$$
\lambda^{\prime}=\max \left(\lambda\left(1-\frac{1}{2} \mu\right)+\mu / 2 k, 1 / k+6 c_{5}(k) \mu+20 \mu\right),
$$

so that indeed $\lambda^{\prime}<\lambda$. We proceed to prove the proposition for $\lambda^{\prime}$.

Write

$$
\delta=\min \left(\varepsilon / 8 \lambda^{\prime}, \varepsilon / 4\right)
$$

and divide the interval $0 \leqslant x \leqslant 1$ into a finite number of subintervals $I$ of length not exceeding $\delta$. If $s$ is large, one of these intervals $I$ will be such that many of the coefficients $a_{1}, \ldots, a_{s}$ are of the type $a_{i}=m^{\alpha_{i}}$ with $\alpha_{i} \in I$. We may therefore suppose without loss of generality that $a_{i} / a_{j} \leqslant m^{\delta}(1 \leqslant i$, $j \leqslant s)$. Similarly we may suppose that $b_{i} / b_{j} \leqslant m^{\delta}(1 \leqslant i, j \leqslant s)$. Put $a=$ $m^{\delta} \max \left(a_{1}, \ldots, a_{s}\right)$ and $b=m^{\delta} \max \left(b_{1}, \ldots, b_{s}\right)$. Let $p_{i}, q_{i}$, respectively, be the largest integers with

$$
a_{i} p_{i}^{k} \leqslant a \quad \text { and } b_{i} q_{i}^{k} \leqslant b \quad(i=1, \ldots, s) .
$$

Now $a / a_{i}>m^{\delta}$, and if $m$ is large (which we may suppose), then $p_{i}>$ $2^{-1 / k}\left(a / a_{i}\right)^{1 / k}$, so that $a_{i} p_{i}^{k} \geqslant \frac{1}{2} a$. Similarly, $b_{i} q_{i}^{k} \geqslant \frac{1}{2} b$.

With $a_{i}^{\prime}=a_{i} p_{i}^{k}, b_{i}^{\prime}=b_{i} q_{i}^{k}$ and $x_{i}=p_{i} x_{i}^{\prime}, y_{i}=q_{i} y_{i}^{\prime}(i=1, \ldots, s)$,

\footnotetext{
${ }^{2} \mathrm{~A}$ reader who finds our nonconstructive argument distasteful should be able to replace it by a constructive one.
} 
becomes

$$
a_{1}^{\prime} x_{1}^{\prime k}+\cdots+a_{s}^{\prime} x_{s}^{\prime k}=b_{1}^{\prime} y_{1}^{\prime k}+\cdots+b_{s}^{\prime} y_{s}^{\prime k} .
$$

If Proposition 1 holds for $\lambda^{\prime}$ and for the particular equation (2.4), then we have a nontrivial nonnegative solution with

$$
\begin{aligned}
\max \left(x_{1}^{\prime}, \ldots, x_{s}^{\prime}, y_{1}^{\prime}, \ldots, y_{s}^{\prime}\right) & \leqslant(\max (a, b))^{\lambda^{\prime}+(\varepsilon / 4)} \\
& \leqslant m^{(1+\delta)\left(\lambda^{\prime}+(\varepsilon / 4)\right)} \leqslant m^{\lambda^{\prime}+(\varepsilon / 2)} .
\end{aligned}
$$

But clearly $a_{i} \geqslant a m^{-2 \delta}$, so that $p_{i} \leqslant p_{i}^{k} \leqslant m^{2 \delta} \leqslant m^{\varepsilon / 2}$, whence $x_{i} \leqslant$ $m^{\lambda^{\prime}+\varepsilon}(i=1, \ldots, s)$, and similarly, $y_{i} \leqslant m^{\lambda^{\prime}+\varepsilon}$, as desired.

(2.4) was special since $\frac{1}{2} a \leqslant a_{i}^{\prime} \leqslant a$ and $\frac{1}{2} b \leqslant b_{i}^{\prime} \leqslant b$. Thus we have the REDUCTION. In proving Proposition 1 for $\lambda^{\prime}$ we may suppose that

$$
\frac{1}{2} a \leqslant a_{i} \leqslant a \text { and } \frac{1}{2} b \leqslant b_{i} \leqslant b \quad(i=1, \ldots, s)
$$

for certain $a, b$.

3. Two cases. In what follows, $h$ will be the integer

$$
h=c_{12}(k, \lambda, \varepsilon)
$$

occurring in Proposition 1, and $s$ will be assumed to be much larger than $h$. Write

$$
\nu=\mu / 2 k
$$

We distinguish two cases.

A. There is a subset of $h$ elements among $a_{1}, \ldots, a_{s}$, say $a_{1}, \ldots, a_{h}$, and there is a subset of $h$ elements among $b_{1}, \ldots, b_{s}$, say $b_{1}, \ldots, b_{h}$, and there are natural integers

$$
p_{1}, \ldots, p_{h}, q_{1}, \ldots, q_{h} \leqslant m^{\nu}
$$

such that

$$
d=\text { g.c.d. }\left(a_{1} p_{1}, \ldots, a_{h} p_{h}, b_{1} q_{1}, \ldots, b_{h} q_{h}\right) \geqslant m^{\mu} \text {. }
$$

In this case put $x_{i}=p_{i} x_{i}^{\prime}, y_{i}=q_{i} y_{i}^{\prime}(i=1, \ldots, h)$ and $x_{h+1}=y_{h+1}$ $=\cdots=x_{s}=y_{s}=0$. After division by $d,(1.4)$ becomes

$$
a_{1}^{\prime} x_{1}^{\prime k}+\cdots+a_{h}^{\prime} x_{h}^{\prime k}=b_{1}^{\prime} y_{1}^{\prime k}+\cdots+b_{h}^{\prime} y_{h}^{\prime k},
$$

where $a_{i}^{\prime}=a_{i} p_{i}^{k} / d$ and $b_{i}^{\prime}=b_{i} q_{i}^{k} / d(i=1, \ldots, h)$. Because of the truth of the proposition for $\lambda$, and by our choice of $h,(3.3)$ has a nontrivial nonnegative solution with

$$
\max \left(x_{1}^{\prime}, \ldots, x_{h}^{\prime}, y_{1}^{\prime}, \ldots, y_{h}^{\prime}\right) \leqslant\left(m^{1+k \nu-\mu}\right)^{\lambda+\varepsilon},
$$

so that

$$
\max \left(x_{1}, \ldots, x_{s}, y_{1}, \ldots, y_{s}\right) \leqslant m^{(1+k \nu-\mu)(\lambda+\varepsilon)+\nu} \leqslant m^{\lambda^{\prime}+\varepsilon} .
$$

We are thus reduced to case 
B. For any $h$ elements, say $a_{1}, \ldots, a_{h}$, among $a_{1}, \ldots, a_{s}$, and for any $h$ elements, say $b_{1}, \ldots, b_{h}$, among $b_{1}, \ldots, b_{s}$, and given (3.2), we have

$$
\text { g.c.d. }\left(a_{1} p_{1}, \ldots, a_{h} p_{h}, b_{1} q_{1}, \ldots, b_{h} q_{h}\right)<m^{\mu} \text {. }
$$

Condition B depends on $h, m, \mu, \nu$, and if $\nu$ is given by (3.1), it is a condition $\mathrm{B}(k, h, m, \mu)$.

Proposition 2. Let $h \geqslant 1, k \geqslant 1$, and

$$
0<\mu<1 / 22 k \text {. }
$$

Let $0<a, b \leqslant m$ and let $a_{1}, \ldots, a_{s}, b_{1}, \ldots, b_{s}$ be integers with

$$
\frac{1}{2} a \leqslant a_{i} \leqslant a, \quad \frac{1}{2} b \leqslant b_{i} \leqslant b \quad(i=1, \ldots, s)
$$

with property $\mathrm{B}(k, h, m, \mu)$. Then if $s \geqslant c_{14}(k, h, \mu)$, the equation

$$
a_{1} x_{1}^{k}+\cdots+a_{s} x_{s}^{k}-\left(b_{1} y_{1}^{k}+\cdots+b_{s} y_{s}^{k}\right)=z
$$

has a solution in nonnegative integers $x_{1}, \ldots, x_{s}, y_{1}, \ldots, y_{s}, z$ with

$$
\max \left(x_{1}, \ldots, x_{s}, y_{1}, \ldots, y_{s}\right) \leqslant m^{(1 / k)+20 \mu}, \quad z \leqslant m^{6 \mu} .
$$

This proposition implies the Inductive Assertion, as we now proceed to show. For let $\lambda, \mu, \lambda^{\prime}, \nu$ be as above, in particular, with (2.2) (whence (3.5)), (2.3), (3.1). We may suppose that we are in the case $\mathrm{B}=\mathrm{B}(k, h, m, \mu)$ with $h=c_{12}(k, \lambda, \varepsilon)$ where $\varepsilon>0$. Suppose that

$$
s=c_{2}(k) c_{14}(k, h, \mu)=n u,
$$

say. After a change of notation, (1.4) becomes

$$
\sum_{i=1}^{n}\left(a_{i 1} x_{i 1}^{k}+\cdots+a_{i u} x_{i u}^{k}-b_{i 1} y_{i 1}^{k}-\cdots-b_{i u} y_{i u}^{k}\right)=0 .
$$

For each $i, 1 \leqslant i \leqslant n$, the coefficients $a_{i 1}, \ldots, a_{i u}, b_{i 1}, \ldots, b_{i u}$ satisfy the conditions of Proposition 2. Hence there are nonnegative $x_{i 1}^{\prime}, \ldots, x_{i u}^{\prime}$, $y_{i 1}^{\prime}, \ldots, y_{i u}^{\prime}$, not all zero, having

$$
a_{i 1} x_{i 1}^{\prime k}+\cdots+a_{i u} x_{i u}^{\prime k}-b_{i 1} y_{i 1}^{\prime k}-\cdots-b_{i u} y_{i u}^{\prime k}=z_{i}
$$

with $\max \left(x_{i 1}^{\prime}, \ldots, x_{i u}^{\prime}, y_{i 1}^{\prime}, \ldots, y_{i u}^{\prime}\right) \leqslant m^{(1 / k)+20 \mu}$ and $0 \leqslant z_{i} \leqslant m^{6 \mu}$. No. Hold it! Keep $0 \leqslant z_{i} \leqslant m^{6 \mu}$ for $i=1, \ldots, n-1$, but ask for $-m^{6 \mu} \leqslant z_{n} \leqslant$ 0 . This is not asking for too much, in view of the symmetry in the + and terms in (3.8). If some $z_{i}=0$, we get a small solution of (3.7) straightaway. If $z_{1}, \ldots, z_{n}$ are each nonzero, then Pitman's estimate (1.2) gives nonnegative $w_{1}, \ldots, w_{n}$, not all zero, with

$$
z_{1} w_{1}^{k}+\cdots+z_{n} w_{n}^{k}=0
$$

having $\max \left(w_{1}, \ldots, w_{n}\right) \leqslant c_{4}(k) m^{6 \mu c_{5}(k)}$. Putting $x_{i j}=w_{i} x_{i j}^{\prime}, y_{i j}=w_{i} y_{i j}^{\prime}(1 \leqslant i$ $<n, 1<j \leqslant u$ ) we obtain a nontrivial solution of (3.7) with

$$
\max \left(x_{i j}, y_{i j}\right)<c_{4}(k) m^{(1 / k)+20 \mu+6 c_{5}(k) \mu}<m^{\lambda^{\prime}}
$$


if $m$ is large. Thus Proposition 1 is true for $\lambda^{\prime}$.

4. Weyl's inequality. Write $e(x)=e^{2 \pi i x}$.

LeMMA 1. Suppose that

$$
|\alpha-u / q|<1 / q^{2} \text { where } q>0,(u, q)=1 .
$$

Then for $\eta>0$,

$$
\left|\sum_{x=1}^{N} e\left(\alpha x^{k}\right)\right| \leqslant c_{15}(k, \eta) N^{1+\eta}\left(N^{-1 / K}+q^{-1 / K}+\left(q / N^{k}\right)^{1 / K}\right)
$$

where $K=2^{k-1}$.

Proof. This is the well-known "Weyl Inequality." See, e.g., [2, Lemma 1].

Corollary. Suppose that $N \geqslant c_{16}(k, \eta), C \geqslant N^{1-(1 / K)+\eta}$ and

$$
\left|\sum_{x=1}^{N} e\left(\alpha x^{k}\right)\right| \geqslant C \text {. }
$$

Then there is a natural

$$
q \leqslant(N / C)^{K} N^{\eta} \text { with }\|\alpha q\| \leqslant(N / C)^{K} N^{\eta-k},
$$

where $\|\cdots\|$ denotes the distance to the nearest integer.

Proof. We have $N^{k-\eta}(C / N)^{K} \geqslant N^{k-\eta-1+K \eta} \geqslant 1$. According to Dirichlet we may pick coprime $q, u$ with

$$
0<q \leqslant N^{k-\eta}(C / N)^{K}
$$

and

$$
|\alpha q-u|=\|\alpha q\| \leqslant(N / C)^{K} N^{\eta-k}
$$

Now

$$
N^{1+(\eta / 2 K)}\left(N^{-1 / K}+\left(q / N^{k}\right)^{1 / K}\right) \leqslant N^{1-(1 / K)+(\eta / 2 K)}+C N^{-\eta / 2 K}
$$

is of smaller order of magnitude than $C$ if $N$ is large. Thus by Lemma 1 (with $\eta / 2 K$ in place of $\eta$ ) we obtain that $N^{1+(\eta / 2 K)} q^{-1 / K} \geqslant c_{17}(k, \eta) C$, whence that $q<(N / C)^{K} N^{\eta}$ if $N$ is large.

5. Application of the Circle Method. Note that it will suffice to prove Proposition 2 for large $m$, say for $m \geqslant c_{18}(k, h, \mu)$. Put

$$
A=\left[b^{1 / k} m^{20 \mu}\right], \quad B=\left[a^{1 / k} m^{20 \mu}\right], \quad H=\left[m^{6 \mu}\right],
$$

where $[\cdots]$ denotes the integer part. If $m$ is sufficiently large, then

$$
A \geqslant 2^{-1 / k} b^{1 / k} m^{20 \mu}, \quad B \geqslant 2^{-1 / k} a^{1 / k} m^{20 \mu} .
$$

Write $Z$ for the number of solutions of (3.6) in integers $x_{1}, \ldots, x_{s}, y_{1}, \ldots, y_{s}$, 
$z$ subject to

$$
1 \leqslant x_{1}, \ldots, x_{s} \leqslant A, \quad 1 \leqslant y_{1}, \ldots, y_{s} \leqslant B, \quad 1 \leqslant z \leqslant H .
$$

We will show that under the assumptions made in the proposition, $Z$ is positive; in fact we will show that $Z$ is at least of the order of magnitude of $H A^{s} B^{s} a^{-1} b^{-1} m^{-20 k \mu}$.

Recall the definition (3.1) of $\nu$ and pick $\eta>0$ with

$$
\eta<1 / 2 K, \quad \eta(1+20 \mu)<\frac{1}{2} \nu,
$$

and pick $s$ so large that

$$
s>(6 K / \nu)+h
$$

All of the parameters $h, \mu, \nu, \eta, s$ will be fixed from now on. We shall employ the 0 -notation or $\ll$ notation with the understanding that the implicit constants may depend on $k, h, \mu, \nu, \eta, s$, but they will be independent of $a_{1}, \ldots, a_{s}, b_{1}, \ldots, b_{s}, a, b, m$. We are going to show that the hypotheses of Proposition 2 imply

$$
Z \gg H A^{s} B^{s} a^{-1} b^{-1} m^{-20 k \mu} .
$$

The number $Z$ will be estimated by the Circle Method. Note that this mẹthod has already been used implicitly, via Pitman's estimate (1.2). We have

$$
Z=\int_{0}^{1} f(\alpha) d \alpha
$$

where

$$
\begin{aligned}
f(\alpha)=\sum_{z=1}^{H} \sum_{x_{1}=1}^{A} \cdots \sum_{x_{s}=1}^{A} \sum_{y_{1}=1}^{B} \cdots & \\
\sum_{y_{s}=1}^{B} e\left(\alpha \left(a_{1} x_{1}^{k}+\cdots+a_{s} x_{s}^{k}\right.\right. & \left.\left.\quad-b_{1} y_{1}^{k}-\cdots-b_{s} y_{s}^{k}-z\right)\right) .
\end{aligned}
$$

We define the major arcs to be the intervals modulo 1 of the type

$$
\mathfrak{M}_{q u}:|\alpha-u / q|<a^{-1} b^{-1} m^{-16 k \mu}
$$

where $q<m^{\mu}$ and $(q, u)=1$.

Lemma 2. Suppose that $|f(\alpha)| \geqslant H A^{s} B^{s} m^{-3}$. Then $\alpha$ lies in a major arc.

Proof. The inequality of the hypothesis implies that

$$
\left|S_{1}(\alpha)\right| \cdots\left|S_{s}(\alpha)\right|\left|T_{1}(\alpha)\right| \cdots\left|T_{s}(\alpha)\right| \geqslant A^{s} B^{s} m^{-3},
$$

where

$$
S_{i}(\alpha)=\sum_{x=1}^{A} e\left(\alpha a_{i} x^{k}\right), \quad T_{i}(\alpha)=\sum_{y=1}^{B} e\left(\alpha b_{i} y^{k}\right)
$$


If, say, $\left|S_{1}(\alpha)\right| \geqslant \cdots \geqslant\left|S_{s}(\alpha)\right|$, then the left-hand side of (5.9) is

$$
\leqslant\left|S_{h}(\alpha)\right|^{s-h+1} A^{h-1} B^{s} \text {, }
$$

so that (5.9) yields

$$
\left|S_{i}(\alpha)\right| \geqslant\left|S_{h}(\alpha)\right| \geqslant A m^{-3 /(s-h+1)}=C, \text { say } \quad(i=1, \ldots, h) .
$$

Observe that

$$
m^{3 /(s-h+1)} \leqslant A^{1 / 6 \mu(s-h+1)} \leqslant A^{(1 / 2 K)} \leqslant A^{(1 / K)-\eta}
$$

by (5.2), (5.4), (5.5). We may apply the Corollary to Lemma 1 to each of the sums $S_{1}(\alpha), \ldots, S_{h}(\alpha)$ to obtain natural numbers $p_{1}, \ldots, p_{h}$ with

$$
p_{i} \leqslant m^{3 K /(s-h+1)} A^{\eta}, \quad\left\|\alpha a_{i} p_{i}\right\| \leqslant m^{3 K /(s-h+1)} A^{\eta-k} \quad(i=1, \ldots, h) .
$$

Using (5.4) and (5.5) again we get

$$
\begin{aligned}
p_{i} & \leqslant m^{\nu} \quad(i=1, \ldots, h), \\
\left\|\alpha a_{i} p_{i}\right\| & \leqslant m^{\nu} A^{-k} \quad(i=1, \ldots, h) .
\end{aligned}
$$

Similarly, after a possible reordering of $b_{1}, \ldots, b_{s}$, there are natural $q_{1}, \ldots, q_{h}$ having

$$
\begin{gathered}
q_{j} \leqslant m^{\nu} \quad(j=1, \ldots, h), \\
\left\|\alpha b_{j} p_{j}\right\| \leqslant m^{\nu} B^{-k} \quad(j=1, \ldots, h) .
\end{gathered}
$$

There are integers $u_{1}, \ldots, u_{h}, v_{1}, \ldots, v_{h}$ with

$$
\left\|\alpha a_{i} p_{i}\right\|=\left|\alpha a_{i} p_{i}-u_{i}\right| \quad(i=1, \ldots, h)
$$

and

$$
\left\|\alpha b_{j} q_{j}\right\|=\left|\alpha b_{j} q_{j}-v_{j}\right| \quad(j=1, \ldots, h) .
$$

Subtracting $a_{i} p_{i}$ times (5.14) from $b_{j} q_{j}$ times (5.12) and observing (5.11), (5.13) and (5.2), we obtain

$$
\begin{aligned}
\left|u_{i} b_{j} q_{j}-v_{j} a_{i} p_{i}\right| & \leqslant b m^{\nu} m^{\nu} A^{-k}+a m^{\nu} m^{\nu} B^{-k} \\
& \leqslant m^{2 \nu-18 \mu k}+m^{2 \nu-18 \mu k}<1
\end{aligned}
$$

if $m$ is sufficiently large. Thus the $2 h$ nonzero vectors $\left(a_{i} p_{i}, u_{i}\right)(i=1, \ldots, h)$ and $\left(b_{j} q_{j}, v_{j}\right)(j=1, \ldots, h)$ are proportional to each other. They are integer multiples of some vector $(q, u)$ where $q>0$ and $q, u$ are coprime. Since $q$ is a common divisor of $a_{1} p_{1}, \ldots, a_{h} p_{h}, b_{1} q_{1}, \ldots, b_{h} q_{h}$, condition (3.4) of case B yields $q<m^{\mu}$. If, say, the vector $\left(a_{i} p_{i}, u_{i}\right)$ is $l_{i}$ times $(q, u)$, then $l_{i} \geqslant \frac{1}{2} a q^{-1}$, whence

$$
\begin{aligned}
|\alpha q-u| & =l_{i}^{-1}\left|\alpha a_{i} p_{i}-u_{i}\right| \leqslant 2 a^{-1} q\left\|\alpha a_{i} p_{i}\right\| \leqslant 2 a^{-1} q m^{\nu} A^{-k} \\
& <2 q a^{-1} b^{-1} m^{\nu-18 k \mu}<q a^{-1} b^{-1} m^{-16 k \mu}
\end{aligned}
$$

if $m$ is large. Thus $\alpha$ lies in $\mathfrak{M}_{q u}$. 
6. The major arcs. Since the major arcs do not overlap, and from Lemma 2, we obtain

$$
Z=\sum_{q<m^{\mu}} \sum_{\substack{u=1 \\(u, q)=1}}^{q} \int_{\mathfrak{M}_{q u}} f(\alpha) d \alpha+O\left(H A^{s} B^{s} m^{-3}\right) .
$$

LEMMA 3. For $\alpha=u / q+\beta \in \mathfrak{M}_{q u}$ we have

$$
S_{i}(\alpha)=q^{-1} \hat{S}_{i}(u / q) I_{i}(\beta)+O\left(m^{5 k \mu}\right) \quad(i=1, \ldots, s)
$$

where

$$
\hat{S}_{i}(u / q)=\sum_{x=1}^{q} e\left(\frac{a_{i} u}{q} x^{k}\right) \text { and } I_{i}(\beta)=\int_{0}^{A} e\left(a_{i} \beta \xi^{k}\right) d \xi .
$$

Proof. Write $x=q y+z$. Then

$$
S_{i}(\alpha)=\sum_{z=1}^{q} e\left(\frac{a_{i} u}{q} z^{k}\right) \sum_{y} e\left(a_{i} \beta(q y+z)^{k}\right),
$$

where the sum over $y$ is over the integers in $1 \leqslant q y+z \leq A$. There will be a certain error if we replace the sum over $y$ by the integral of $e\left(a_{i} \beta(q \zeta+z)^{k}\right)$ with respect to $\zeta$, with the range of integration given by $0 \leqslant q \zeta+z \leqslant A$. The function

$$
g(\zeta)=e\left(a_{i} \beta(q \zeta+z)^{k}\right)
$$

has

$$
\left|g^{\prime}(\zeta)\right| \leqslant q a_{i}|\beta| A^{k-1}, \quad|g(\zeta)| \leqslant 1
$$

in this range, and this range is an interval of length $A / q$. Therefore

$$
\begin{gathered}
\left|\sum_{y} e\left(a_{i} \beta(q y+z)^{k}\right)-\int e\left(a_{i} \beta(q \beta+z)^{k}\right) d \zeta\right| \\
\leqslant(A / q)\left(q a_{i}|\beta| A^{k-1}\right)+3 \leqslant A^{k} a|\beta|+3 \\
<A^{k} a a^{-1} b^{-1} m^{-16 k \mu}+3<m^{4 k \mu}+3 .
\end{gathered}
$$

Taking the sum over $z$ in (6.3) we get

$$
S_{i}(\alpha)=\sum_{z=1}^{q} e\left(\frac{a_{i} u}{q} z^{k}\right) \int e\left(a_{i} \beta(q \zeta+z)^{k}\right) d \zeta+O\left(m^{5 k \mu}\right) .
$$

The change of variables $\xi=q \zeta+z$ yields the desired result.

In analogy to Lemma 3 we obtain

$$
T_{i}(\alpha)=q^{-1} \hat{T}_{i}(u / q) J_{i}(\beta)+O\left(m^{5 k \mu}\right) \quad(i=1, \ldots, s)
$$

where $\hat{T}_{i}, J_{i}$ are defined in the obvious way. 
LEMMA 4. If $\mathfrak{M}$ is the totality of the major arcs, then

$$
\begin{aligned}
\int_{\mathfrak{M}} f(\alpha) d \alpha= & A^{s} B^{s} a^{-1} b^{-1} m^{-20 k \mu} \subseteq\left(m^{\mu}, H\right) \Im\left(m^{4 k \mu}\right) \\
& +O\left(H A^{s} B^{s} a^{-1} b^{-1} m^{-22 k \mu}\right),
\end{aligned}
$$

where the "singular series"

$$
\begin{aligned}
& S\left(m^{\mu}, H\right)=\sum_{z=1}^{H} \sum_{q<m^{\mu}} \sum_{\substack{u=1 \\
(q, u)=1}}^{q} q^{-2 s} \hat{S}_{1}\left(\frac{u}{q}\right) \cdots \\
& \hat{S}_{s}\left(\frac{u}{q}\right) \hat{T}_{1}\left(\frac{u}{q}\right) \cdots \hat{T}_{s}\left(\frac{u}{q}\right) e\left(-\frac{u}{q} z\right),
\end{aligned}
$$

and the "singular integral"

$$
\Im\left(m^{4 k \mu}\right)=\int_{|\beta|<m^{4 k \mu}} \prod_{i=1}^{s}\left(\int_{0}^{1} e\left(\rho_{i} \xi_{i}^{k} \beta\right) d \xi_{i}\right) \prod_{j=1}^{s}\left(\int_{0}^{1} e\left(-\sigma_{i} \zeta_{i}^{k} \beta\right) d \zeta\right) d \beta,
$$

for certain constants $\rho_{1}, \ldots, \rho_{s}, \sigma_{1}, \ldots, \sigma_{s}$ in the interval $\left[\frac{1}{4}, 1\right]$.

Proof. The integral in question is

$$
\sum_{z=1}^{H} \sum_{q<m^{\mu}} \sum_{\substack{u=1 \\(q, u)=1}}^{q} \int_{\mathfrak{M}_{q u}} S_{1}(\alpha) \ldots S_{s}(\alpha) T_{1}(\alpha) \ldots T_{s}(\alpha) e(-z \alpha) d \alpha .
$$

If $\alpha=u / q+\beta$ lies in $\mathfrak{M}_{q u}$, then Lemma 3 and the trivial estimates $\left|I_{i}(\beta)\right| \leqslant$ $A,\left|J_{i}(\beta)\right| \leqslant B$ yield

$$
\begin{aligned}
& S_{1}(\alpha) \cdots S_{s}(\alpha) T_{1}(\alpha) \cdots T_{s}(\alpha) \\
& =q^{-2 s} \hat{S}_{1}\left(\frac{u}{q}\right) \cdots \hat{S}_{s}\left(\frac{u}{q}\right) \hat{T}_{1}\left(\frac{u}{q}\right) \cdots \\
& \qquad \hat{T}_{s}\left(\frac{u}{q}\right) I_{1}(\beta) \cdots I_{s}(\beta) J_{1}(\beta) \cdots J_{s}(\beta) \\
& \quad+O\left(A^{s} B^{s} \max \left(m^{5 k \mu} A^{-1}, m^{5 k \mu} B^{-1}\right)\right) .
\end{aligned}
$$

The error term here is $O\left(A^{s} B^{s} m^{-15 k \mu}\right)$, and since $\mathfrak{M}_{q u}$ is of length $2 a^{-1} b^{-1} m^{-16 k \mu}$, the integral over $\mathfrak{M}_{q u}$ in (6.5) is

$$
\begin{array}{r}
q^{-2 s} \hat{S}_{1}\left(\frac{u}{q}\right) \cdots \hat{T}_{s}\left(\frac{u}{q}\right) e\left(-\frac{u}{q} z\right) \int_{|\beta|<a^{-1} b^{-1} m^{-16 k \mu}} I_{1}(\beta) \\
\cdots J_{s}(\beta) e(-\beta z) d \beta+O\left(A^{s} B^{s} a^{-1} b^{-1} m^{-30 k \mu}\right) .
\end{array}
$$


In the integral in (6.6) we replace $e(-\beta z)$ by 1 . The error is $\ll A^{s} B^{s} z\left(a^{-1} b^{-1} m^{-16 k \mu}\right)^{2} \ll A^{s} B^{s} H a^{-2} b^{-2} m^{-32 k \mu} \ll A^{s} B^{s} a^{-1} b^{-1} m^{-25 k \mu}$. Thus the integral over $\mathfrak{M}_{q u}$ in (6.5) is

$$
\begin{array}{r}
q^{-2 s} \hat{S}_{1}\left(\frac{u}{q}\right) \cdots \hat{T}_{s}\left(\frac{u}{q}\right) \int_{|\beta|<a^{-1} b^{-1} m^{-16 k \mu}} I_{1}(\beta) \cdots \\
J_{s}(\beta) d \beta+O\left(A^{s} B^{s} a^{-1} b^{-1} m^{-25 k \mu}\right) .
\end{array}
$$

To evaluate the integral in (6.7), put $\xi_{i}=A \xi_{i}^{\prime}(i=1, \ldots, s), \zeta_{i}=B \zeta_{i}^{\prime}$ $(i=1, \ldots, s)$ and $\beta=a^{-1} b^{-1} m^{-20 k \mu} \beta^{\prime}$. Then

$$
a_{i} \beta \xi_{i}^{k}=\left(a_{i} A^{k} / a b m^{20 k \mu}\right) \beta^{\prime} \xi_{i}^{\prime k}=\rho_{i} \beta^{\prime} \xi_{i}^{\prime k} \quad(i=1, \ldots, s),
$$

say, where by (5.2), $\frac{1}{4} \leqslant \rho_{i} \leqslant 1$. Similarly, $-b_{i} \beta \zeta_{i}^{k}=-\sigma_{i} \beta^{\prime} \zeta_{i}^{\prime k}$. The integral in (6.7) becomes

$$
A^{s} B^{s} a^{-1} b^{-1} m^{-20 k \mu} \Im\left(m^{4 k \mu}\right)
$$

and the integral over $\mathfrak{M}_{q u}$ in (6.5) turns out to be

$$
\begin{aligned}
A^{s} B^{s} a^{-1} b^{-1} m^{-20 k \mu} q^{-2 s} \hat{S}_{1}(u / q) \cdots \hat{T}_{s}(u / q) e(-u z / q) \Im\left(m^{4 k \mu}\right) \\
+O\left(A^{s} B^{s} a^{-1} b^{-1} m^{-25 k \mu}\right) .
\end{aligned}
$$

Taking the sum over $z, q, u$ in (6.5) we obtain Lemma 4 .

7. The singular integral. We have

$$
\begin{aligned}
\int_{0}^{1} e\left(\rho_{i} \xi_{i}^{k} \beta\right) d \xi_{i} & =k^{-1} \rho_{i}^{-1 / k} \int_{0}^{\rho_{i}} \varphi_{i}^{-1+(1 / k)} e\left(\varphi_{i} \beta\right) d \varphi_{i} \\
& =k^{-1}\left(\rho_{i} \beta\right)^{-1 / k} \int_{0}^{\rho_{i} \beta} \varphi_{i}^{-1+(1 / k)} e\left(\varphi_{i}\right) d \varphi_{i} .
\end{aligned}
$$

The last integral is bounded as a function of the upper limit of integration so that the integral on the left is $\ll \beta^{-1 / k}$. It follows that as a function of $m$,

$$
\mathfrak{\Im}\left(m^{4 k \mu}\right)=\mathfrak{\Im}(\infty)+o(1),
$$

where $\mathfrak{\Im}(\infty)$ is as $\mathfrak{\Im}\left(\mathrm{m}^{4 k \mu}\right)$, but with the integral over $\beta$ extended over the real line. Using the middle expression in (7.1) we get

$$
\begin{aligned}
\Im(\infty)= & k^{-2 s}\left(\rho_{1} \cdots \sigma_{s}\right)^{-1 / k} \\
& \cdot \int_{-\infty}^{\infty} d \beta \int_{0}^{\rho_{1}} d \varphi_{1} \cdots \int_{0}^{\sigma_{s}} d \psi_{s}\left(\varphi_{1} \cdots \varphi_{s} \psi_{1} \cdots \psi_{s}\right)^{-1+(1 / k)} \\
& \cdot e\left(\left(\varphi_{1}+\cdots+\varphi_{s}-\psi_{1}-\cdots-\psi_{s}\right) \beta\right) \\
= & k^{-2 s}\left(\rho_{1} \cdots \sigma_{s}\right)^{-1 / k} \\
& \cdot \lim _{\omega \rightarrow \infty} \int_{0}^{\rho_{1}} d \varphi_{1} \cdots \int_{0}^{\sigma_{s}} d \psi_{s}\left(\varphi_{1} \cdots \varphi_{s} \psi_{1} \cdots \psi_{s}\right)^{-1+(1 / k)} \\
& \cdot \frac{\sin 2 \pi \omega\left(\varphi_{1}+\cdots+\varphi_{s}-\psi_{1} \cdots \cdots-\psi_{s}\right)}{\pi\left(\varphi_{1}+\cdots+\varphi_{s}-\psi_{1}-\cdots-\psi_{s}\right)}
\end{aligned}
$$


as in [2, p. 27]. Continuing as in [2] we get

$$
\Im(\infty)=k^{-2 s}\left(\rho_{1} \cdots \sigma_{s}\right)^{-1 / k} \lim _{\omega \rightarrow \infty} \int_{-s}^{s} \Omega(\omega) \frac{\sin 2 \pi \omega u}{\pi u} d \omega,
$$

where

$$
\begin{aligned}
\Omega(\omega)= & \int_{0}^{\rho_{1}} d \varphi_{1} \cdots \int_{0}^{\rho_{s}} d \varphi_{s} \int_{0}^{\sigma_{1}} d \psi_{1} \cdots \int_{0}^{\sigma_{s-1}} d \psi_{s-1} \\
& \\
& \cdot\left(\varphi_{1} \cdots \varphi_{1}+\cdots+\varphi_{s}-\psi_{1}-\cdots-\psi_{s-1}<u+\sigma_{s}\right. \\
& \left.\quad-\psi_{s-1}\left(\varphi_{1}+\cdots+\varphi_{s-1}-u\right)\right)^{-1+(1 / k)} .
\end{aligned}
$$

The limit in (7.3) equals

$$
\begin{aligned}
\Omega(0) & \geqslant \int_{0}^{1 / 4} d \varphi_{1} \cdots \int_{0<\varphi_{1}+\cdots+\varphi_{s}-\psi_{1}-\cdots-\psi_{s-1}<1 / 4}^{1 / 4} d \varphi_{0}^{1 / 4} d \psi_{1} \cdots \int_{0}^{1 / 4} d \psi_{s-1} \\
& \cdot\left(\varphi_{1} \cdots \varphi_{s} \psi_{1} \cdots \psi_{s-1}\left(\varphi_{1}+\cdots+\varphi_{s}-\psi_{1}-\cdots-\psi_{s-1}\right)\right)^{-1+(1 / k)} \\
& \gg 1 .
\end{aligned}
$$

Combining our estimates we find that for $m$ sufficiently large,

$$
\Im\left(m^{4 k \mu}\right) \gg 1 \text {. }
$$

8. The singular series.

$$
\begin{aligned}
S\left(m^{\mu}, H\right)=\sum_{z=1}^{H} \sum_{q<m^{\mu}} \sum_{\substack{u=1 \\
(u, q)=1}}^{q} \sum_{x_{1}=1}^{q} \cdots \\
\\
\sum_{y_{s}=1}^{q} q^{-2 s} e\left(\frac{u}{q}\left(a_{1} x_{1}^{k}+\cdots-b_{s} y_{s}^{k}-z\right)\right) .
\end{aligned}
$$

The summands with $q=1$ give the contribution $H$.

When $q>1$,

$$
\sum_{z=1}^{H} e(-u z / q) \ll q,
$$

so that the summands with fixed $q>1$ contribute $\ll q^{2}$. Since $\Sigma q^{2}$ over $q<m^{\mu}$ is $\ll m^{3 \mu}$, we obtain

$$
\Im\left(m^{\mu}, H\right)=H+O\left(m^{3 \mu}\right) \gg H .
$$

9. Conclusion. Combining (6.1), Lemma 4, (7.4) and (8.1) we get

$$
Z \gg H A^{s} B^{s} a^{-1} b^{-1} m^{-20 k \mu}+O\left(H A^{s} B^{s} m^{-3}+H A^{s} B^{s} a^{-1} b^{-1} m^{-22 k \mu}\right) .
$$

Since $m^{3}>a b m \geqslant a b m^{22 k \mu}$ by (3.5), the error term here is smaller than the main term, and (5.6) follows. 


\section{REFERENCES}

1. B. J. Birch, Small zeros of diagonal forms of odd degree in many variables, Proc. London Math. Soc. (3) 21 (1970), 12-18.

2. H. Davenport, Analytic methods for diophantine equations and diophantine inequalities, Lecture Notes, Univ. of Michigan, 1962.

3. H. Davenport and D. Lewis, Homogeneous additive equations, Proc. Roy. Soc. Ser. A 274 (1963), 443-460.

4. A. E. Gel'fond and Yu. V. Linnik, Elementary methods in analytic number theory, Rand McNally, Chicago, 1965.

5. Yu. V. Linnik, An elementary solution of Waring's problem by Schnirelman's method, Mat. Sb. 12 (54) (1943), 225-230. (Russian)

6. J. Pitman, Bounds for solutions of diagonal equations, Acta Arith. 19 (1971), 223-247.

7. H. P. Schlickewei, On indefinite diagonal forms in many variables, J. Reine Angew Math. (to appear).

Department of Mathematics, University of Colorado, Boulder, Colorado 80309 